\title{
Clotting of Hemodialysis Access in Patients with COVID-19 in an Inner-City Hospital
}

\author{
Jie Ouyang ${ }^{a, b}$ Siddhartha Bajracharya ${ }^{a, b}$ Sabu John ${ }^{a, b}$ John Wagner ${ }^{a}$ \\ Jiehui Xuc ${ }^{c}$ Yiming Luo $^{d}$ Mariah Thaxton ${ }^{a}$ Moro Salifu ${ }^{a, b}$ Ernie Yap ${ }^{a, b}$ \\ Mary Mallappallila, b
}

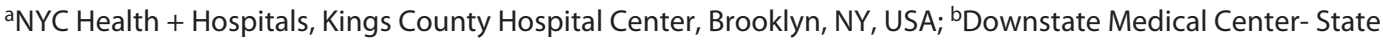
University of Brooklyn, Brooklyn, NY, USA; 'Division of Biostatistics, New York University, New York, NY, USA;

${ }^{\mathrm{d}}$ Rheumatology, National Institute of Arthritis and Musculoskeletal and Skin Disease, Bethesda, MD, USA

\section{Keywords}

Coronavirus disease 2019 - Coronavirus · Hemodialysis ·

Access $\cdot$ Catheter $\cdot$ Clotting

\begin{abstract}
Background: An increased incidence of thrombotic complications in patients with coronavirus disease 2019 (COVID-19) has been reported. Severe acute kidney injury (AKI) is one of the major clinical manifestations of COVID-19 with the need for renal replacement therapy. It was observed that hemodialysis (HD) accesses tended to thrombose more often in the COVID-19 population than in non-COVID-19 patients. We hypothesize that the hypercoagulable state of COVID-19 is associated with higher incidence of access clotting. Method: In this retrospective single-centered study at Kings County Hospital in New York City, 1,075 patients with COVID-19 were screened, and 174 patients who received HD from January 3, 2021 to May 15, 2020 were enrolled to examine the risk factors of dialysis access clotting in patients with $\mathrm{CO}$ VID-19. Results: Of the 174 patients, 109 (63\%) were COVID-19 positive. 39 (22.6\%) patients had dialysis access clotting at least once during their hospitalization, and they had significantly higher body mass index (BMI) ( $p=0.001)$, high-
\end{abstract}

er rates of COVID-19 ( $p=0.015)$, AKI $(p<0.001)$, higher platelet counts $(p=0.029)$, higher lactate dehydrogenase levels $(p=0.009)$, and lower albumin levels $(p=0.001)$ than those without access malfunctions. Low albumin levels $(p=0.008)$, $\operatorname{AKI}(p=0.008)$, and high BMI $(p=0.018)$ were risk factors associated with HD access clotting among COVID-19 patients. Conclusion: Patients with COVID-19 who receive HD for AKI with high $\mathrm{BMI}$ are at a higher risk of clotting their $\mathrm{HD}$ access.

() 2021 S. Karger AG, Basel

\section{Introduction}

Early reports of complications in patients with coronavirus disease 2019 (COVID-19) suggested an increased incidence of thrombotic events $[1,2]$; among such patients admitted to intensive care units (ICUs), thrombotic events were $31 \%$ higher than expected [3]. Multiple mechanisms were hypothesized, including endothelial injury [4], immunothrombosis [5], and coagulation abnormalities [6]. Although the precise mechanisms remain unclear, many studies of COVID-19 patients have observed a hypercoagulable state [7], which leads to increased incidence of both arterial and venous thromboses, as well as organ is- karger@karger.com www.karger.com/nef
(C) 2021 S. Karger AG, Basel

Karger ${ }^{\prime}=$
Correspondence to:

Mary Mallappallil, mary.mallappallil@ nychhc.org 
chemia. Coagulopathy is independently associated with increased mortality in COVID-19 patients [6]. Cui et al. [8] noted that the incidence of venous thromboembolism (VTE) in patients with COVID-19 pneumonia was $25 \%$ $(n=20 / 81)$, with a high mortality in this subgroup. Most studies indicated some mortality benefit of heparin use in the patients with COVID-19, at least in those with more severe disease $[6,9,10]$.

Acute kidney injury (AKI) is one of the major clinical manifestations of COVID-19. Patients with severe AKI require renal replacement therapy (RRT), typically through hemodialysis (HD) or continuous RRT; we noticed that the HD access tended to thrombose more often in the COVID-19 population in our practice, a finding that was also revealed recently by Karina et al. [11]. In order to understand this phenomenon, we present this study to assess the increased risk of clotting dialysis access in this population. We hypothesized that the hypercoagulable state of COVID-19 is associated with a higher incidence of $\mathrm{HD}$ access clotting. We expect to determine potential risk factors of hypercoagulability and the tendency of $\mathrm{HD}$ access clotting by examining variable characteristics of patients with and without COVID-19 who required RRT for either severe AKI or end-stage renal disease (ESRD) during the early phase of the COVID-19 pandemic.

\section{Methods}

This is a retrospective single-center study at Kings County Hospital (KCH), an inner-city hospital in New York City. KCH had 45 ICU beds that were expanded to approximately 150 ICU beds for COVID-19. This study was approved by the Institutional Review Board at SUNY Downstate Medical Center and New York City Health and Hospital Corporation.

3,665 patients were hospitalized in $\mathrm{KCH}$ between March 1st and May 15th, 2020, of whom 1,075 had laboratory-confirmed COVID-19 during their admission. We extracted medical record data from the charts of those who required HD because of either severe AKI or ESRD during this time period.

We identified potential study participants using the Epic EHR analytics platform SlicerDicer. The inclusion criteria were as follows: 1 . Patients who were older than 18 years; 2 . patients who were admitted to KCH from March 1st to May 15th; 3. patients who had received HD. Patients who met the inclusion criteria were automatically enrolled with a full waiver of written consent approved by both SUNY Downstate and New York City Health and Hospital Corporation institutional review boards.

These patients' charts were manually reviewed, including notes, medications, records of interventional procedures and $\mathrm{HD}$, and laboratory results. We examined the incidence of $\mathrm{HD}$ access malfunction and patient characteristics to discover risk factors of dialysis access clotting. The primary outcome was the clotting of
HD access in those who were diagnosed with COVID-19 and those without COVID-19. The diagnosis of COVID-19 was by laboratory RNA polymerase chain reaction confirmation and/or clinical manifestation of viral pneumonia with high levels of inflammatory factors and severe hypoxia. HD access failure was diagnosed by persistent significant decrease in blood flow preventing continuing HD, regardless of repositioning, change of the tunneled or nontunneled catheter, or the use of tissue plasminogen activator in the catheter. Arteriovenous fistula (AVF) or graft (AVG) failure was diagnosed by a significant decrease in blood flow that led to HD cessation, a new tunneled/nontunneled catheter placement, or other clinical findings indicative of AVF/AVG malfunctioning. All malfunctioned dialysis accesses were examined and reported by dialysis nurses, confirmed by nephrology fellows, and attending physicians.

\section{Statistical Analysis}

We analyzed the descriptive statistics of the included patients' demographic features, medical history, in-hospital laboratory results, treatments administered, and outcomes during current admission. We compared these variables between patients with $\mathrm{HD}$ access clotting and those without using Fisher's exact test for categorical variables or Wilcoxon's rank-sum test for continuous variables. We chose these nonparametric tests as most groups of the dataset were not normally distributed.

To determine clotting risk factors within the COVID-19-positive patients' group, we selected variables that were significantly different in the 2 subgroups in Table 1 for the logistic regression test using the stepwise strategy. We removed D-dimer from the analysis in order to maximize the sample size due to a large number of missing data of D-dimer values. 109 patients with COVID-19 were included in this analysis. The statistical analysis was all conducted using R software (version 3.6.3).

\section{Results}

We compared data during the early phase of the pandemic to a historical control that was 2 months earlier in 2020 and noticed a significantly increased rate of AKI and incidence of dialysis access failure. From December 16th to February 29th, 2020, there were totally 97 admitted patients who received $\mathrm{HD}$, with 3 patients $(3.1 \%)$ being diagnosed with AKI that required $\mathrm{HD}$, and 8 out of these 97 patients (8.2\%) developed dialysis access malfunctioning. While 174 patients received HD from March 1st to May 15th, 2020, ninety-two patients received RRT (mostly intermittent HD) because of severe AKI (52.0\%), and 122 patients (70.1\%) received dialysis via tunneled or nontunneled catheters. Thirty-nine out of 174 patients (22.6\%) experienced dialysis access clotting at least once during hospitalization. Eight of the 39 patients $(20.5 \%)$ had ESRD, and 2 of them (25\%) experienced AVF and AVG failure. Seventy-four patients $(42.5 \%)$ had died by the time of data collection in July 2020. 
Table 1. Characteristics of patients received hemodialysis between March 1 to May 15, 2020

\begin{tabular}{|c|c|c|c|c|c|}
\hline \multicolumn{6}{|l|}{ Demographic } \\
\hline African American & $148(85.1)$ & $33(84.6)$ & $119(85.2)$ & 0.832 & 0 \\
\hline $\mathrm{BMI}$ & $29.4(7.7)$ & $33.1(8.6)$ & $28.3(7.1)$ & 0.001 & 4 \\
\hline \multicolumn{6}{|l|}{ Medical history } \\
\hline Diabetes & $112(64.4)$ & $27(69.2)$ & $85(63.0)$ & 0.57 & 0 \\
\hline Hypertension & $149(85.6)$ & $31(79.5)$ & $118(87.4)$ & 0.298 & 0 \\
\hline Asthma and/or COPD & $24(13.8)$ & $3(7.7)$ & $21(15.6)$ & 0.294 & 0 \\
\hline Cancer & $12(6.9)$ & $3(7.7)$ & $9(6.7)$ & 0.733 & 0 \\
\hline D-dimer levels $(\times 1,000)$ & $13.6(2.0)$ & $15.5(1.9)$ & $12.9(2.0)$ & 0.048 & 58 \\
\hline Lowest albumin levels & $2.67(0.76)$ & $2.34(0.62)$ & $2.77(0.78)$ & 0.001 & 0 \\
\hline Platelet $\times 1,000$ & $219.8(104.4)$ & $237.1(86.7)$ & $214.8(108.8)$ & 0.029 & 0 \\
\hline PT & $14.23(5.27)$ & $13.29(1.98)$ & $14.54(5.95)$ & 0.2 & 29 \\
\hline INR & $1.25(0.46)$ & $1.14(0.21)$ & $1.28(0.51)$ & 0.132 & 29 \\
\hline LDH & $744(543)$ & $900(540)$ & $689(536)$ & 0.009 & 40 \\
\hline Ferritin $\times(100)$ & $15.6(7.7)$ & $16.7(8.8)$ & $15.2(7.2)$ & 0.496 & 28 \\
\hline Mechanical ventilation & $48(27.6)$ & $5(12.8)$ & $43(31.9)$ & 0.024 & 0 \\
\hline Received heparin therapy via any route & $84(48.3)$ & $30(76.9)$ & $54(40.0)$ & $<0.001$ & 0 \\
\hline Hydroxychloroquine therapy & $96(55.2)$ & $28(71.8)$ & $68(50.4)$ & 0.019 & 0 \\
\hline \multicolumn{6}{|l|}{ Outcome } \\
\hline Mortality & $74(42.5)$ & $19(48.7)$ & $55(40.7)$ & 0.583 & 0 \\
\hline
\end{tabular}

BMI, body mass index; COPD, chronic obstructive pulmonary disease; COVID-19, coronavirus disease 2019; PT, prothrombin time; INR, international normalized ratio; LDH, lactate dehydrogenase; AKI, acute kidney injury.

Bivariate correlation of all variables was analyzed. COVID-19 diagnosis and hydroxychloroquine use were strongly correlated as expected ( $\mathrm{rho}=0.713, p<0.01$ ).

As shown in Table 1, the majority of the research subjects were African American (85.1\%), male (62.1\%), older (62.6 \pm 12.7$)$, and slightly overweighted (29.4 \pm 7.7$)$. The most common medical history included hypertension (85.6\%) and diabetes mellitus (64.4\%). 109 patients (62.6\%) manifested COVID-19 infection. When those whose HD access clotted were compared to the patients who had no dialysis access failure, a higher body mass in$\operatorname{dex}(\mathrm{BMI})(p=0.001)$, a higher rate of COVID-19 infection $(p=0.015)$, and more AKI cases $(p<0.001)$ were noticed. Higher D-dimer levels (although 58 patients had missing data) $(p=0.048)$, lower albumin levels $(p=0.001)$, higher platelet levels $(p=0.029)$, higher lactate hydrogenase levels $(p=0.009)$, less mechanical ventilation usage
Table 2. Correlations between AKI and lowest albumin levels

\begin{tabular}{ccc}
\hline & Coefficient & $p$ value \\
\hline AKI and lowest albumin levels & -0.651 & 0.000 \\
\hline AKI, acute kidney injury. & & \\
\hline
\end{tabular}

( $p=0.024)$, higher chance of heparin $(p<0.001)$, and hydroxychloroquine therapy $(p=0.019)$ were also associated with an increased risk of HD access failure. There was no significant difference of the mortality rate in these 2 groups.

In the COVID-19 patients' group, when the potential mechanisms of the hypercoagulable state of COVID-19 were examined, very interesting results were noticed in 
Table 3. The potential risk factors for clotting in COVID-19 patients

\begin{tabular}{llllll}
\hline & Coefficient & Adjusted odds & $95 \% \mathrm{Cl}$ & & $p$ value \\
\hline BMI & 0.07 & 1.07 & 1.01 & 1.13 & 0.018 \\
Hypertension & -1.17 & 0.31 & 0.10 & 0.99 & 0.046 \\
Platelet*100 & 0.43 & 1.53 & 0.94 & 2.48 & 0.085 \\
LDH*1,000 $^{*}$ AKI & 0.15 & 1.16 & 0.52 & 2.64 & 0.697 \\
& 1.55 & 4.72 & 1.60 & 16.30 & 0.008 \\
\hline
\end{tabular}

AKI, acute kidney injury; LDH, lactate dehydrogenase; COVID-19, coronavirus disease 2019; BMI, body mass index. * When AKI is replaced by lowest albumin levels, the analysis yields the same statistically significant risk factors, and the $p$ value for lowest albumin levels is also 0.008 .
Tables 2 and 3. AKI and low albumin levels were significant risk factors when they fit in the model separately; however, they were not significant when both of them were included in the same model. Analysis confirmed significant correlation between AKI and low albumin levels, which explains this interesting finding. Meanwhile, higher BMI and history of hypertension remained significant, suggesting association with developing HD access clotting.

\section{Discussion}

This retrospective single-center study found a significantly increased rate of clotting of HD access in hospitalized patients who had COVID-19. Clotting was not only limited to catheters but also affected AVF and AVG. Strikingly, within the COVID-19 patients' group, severe AKI requiring HD as well as higher BMI were independent factors that strongly associated with access malfunction. Patients who presumably were on maintenance $\mathrm{HD}$ for ESRD via tunneled catheter or AVF/AVG had a lower rate of access complications; notably, only 2 patients with AVF and AVG had access malfunction (which ultimately required tunneled dialysis catheter placement after a failed intervention). These findings are consistent with other data in patients with COVID-19 and renal failure that have reported a high rate of clot formation in the extracorporeal circuit used for continuous RRT and a 50\% shortened circuit patency $[12,13]$.

In critically ill patients with AKI requiring $\mathrm{HD}$ in prior studies before the pandemic, rates of thrombotic catheter malfunction were quite low. The incidence rate of nontunneled HD access complications in our historical data is similar to that of these studies $[14,15]$. Our analysis suggests that among all study participants who received HD, COVID-19 specifically but not AKI resulted in thrombotic dialysis complications; Meanwhile, several prior studies $[16,17]$ investigating thrombotic events in COVID-19 patients did not report acute renal dysfunction as a significant risk factor; only one small study of large vessel thromboses in patients with COVID-19 found that 10 out of 21 such patients had AKI. Although the mechanism is not yet clarified, COVID-19 was a major risk factor for the access clotting in our analysis, which was also noted in other studies [6]. The reasons why COVID-19 patients with AKI required dialysis had a higher rate of access malfunction than ESRD patients with COVID-19 are unclear [18]. While studies investigated only native vessel thromboses, we had a more inclusive definition of clotting events effecting dialysis catheters. We speculate that the apparent hypercoagulable state in patients with COVID-19 may particularly effect intravascular foreign bodies such as dialysis catheters.

As we report 2 cases of AVF and AVG dysfunction in this time period, a survey of 255 ESRD patients with COVID-19 found 17 cases of AVF and AVG thromboses, suggesting that while the risk is reduced in ESRD patients, it is still quite substantial [19].

In our analysis, we noted a higher rate of administration of heparin including intra-catheter, intra-venous, and subcutaneous administration in the group of patients who had $\mathrm{HD}$ access clotting. It is more likely that the hyper-coagulopathy or HD access clotting was noticed before active anticoagulation therapy was initiated in this group.

Interestingly, a recent multicenter study noted that early initiation of anticoagulation did not improve survival in those critically ill patients with COVID-19 [20], although autopsy data suggest immune-thrombotic dysregulation with microthrombi formation in those with COVID 19 critical illness [5]. A possible explanation may be that the prophylactic dose of heparin might not be adequate.

Obesity has been associated with worse outcomes with AKI in critical illness [21-23], and it was included in a 
COVID-19 scoring system for predicting worse outcomes [24]. In our analysis, higher BMI persisted in contributing to access clotting. It is unclear how it contributed to the worse outcome or hypercoagulability among patients on $\mathrm{HD}$, yet it remains important to reveal the mechanism as it is a modifiable risk factor and a potential indication for aggressive anticoagulation therapy, which can possibly improve patient outcomes.

In our study, hypoalbuminemia is a factor being colinear with AKI which was associated with HD access malfunction among patients with COVID-19. Hypoalbuminemia has been connected with worse outcomes in patients with COVID-19 in a meta-analysis [25] and in studies of critically ill patients prior to the pandemic. Low albumin may be predictive of development of mortality following AKI [26]. Studies have suggested that in the general population, hypoalbuminemia may be associated with increased risk for VTE $[27,28]$. In Brosnahan et al.'s [16] investigation of patients with COVID-19, similar association between low albumin and increased risk for a thrombotic event was also noticed. A recent pilot study [29] also hypothesized albumin supplementation may be beneficial in reducing the risk of hypercoagulability in patients with COVID-19; unfortunately, no clear conclusions could be drawn due to the small sample size. These results and hypotheses suggest a research opportunity on the mechanism of hypercoagulability among COVID-19 patients, especially those who develop severe AKI, and a potential treatment by improving the albumin level.

This is one of the very few studies that evaluated increased incidence of HD access clotting in patients with COVID-19. The limitations of the presenting study include that the majority of enrolled patients are of a single race/ethnicity; it is a retrospective chart review from a single-center.

\section{Conclusion}

In conclusion, patients with COVID-19 are at a higher risk of clotting their $\mathrm{HD}$ access. Having a higher BMI is associated with a higher risk of HD access clotting regardless of COVID-19. Albumin played an important role in HD access clotting among patients with COVID-19. The incidence of access clotting is higher among patients who suffered from severe AKI caused by COVID-19. Further studies are needed to determine the mechanism of hypercoagulopathy in order to effectively prevent thromboses and decrease mortality of COVID-19.

\section{Statement of Ethics}

This is a retrospective study. All data were collected via chart review. A full waiver of written or oral consent was granted by SUNY Downstate Healthy Science University IRB and NYC Health + Hospital IRB.

\section{Conflict of Interest Statement}

None of the authors have any disclosures that are relevant to the study and manuscript.

\section{Funding Sources}

This study and manuscript are not sponsored by any private or public funding.

\section{Author Contributions}

All listed authors meet at least 4 of the following criteria for authorship: conception or design of the work, data collection, data analysis and interpretation, drafting the article, critical revision of the article, final approval of the version to be published, and ensuring the accuracy or integrity of the research work.

\section{Data Availability Statement}

The de-identified data used for analysis in this manuscript are saved in the NYCHHC KCH server. It will not be publicly available due to the NYCHHC IRB's requirement, but it can be provided via an encrypted email for review upon reasonable request. Further inquires can be directed to the corresponding author.

\section{References}

1 Bilaloglu S, Aphinyanaphongs Y, Jones S, Iturrate E, Hochman J, Berger JS. Thrombosis in hospitalized patients with COVID-19 in a New York City health system. JAMA. 2020; 324(8):799-801.

2 Connors JM, Levy JH. COVID-19 and its implications for thrombosis and anticoagulation. Blood. 2020 Jun 4;135(23):2033-40.

3 Klok FA, Kruip MJHA, van der Meer NJM, Arbous MS, Gommers DAMPJ, Kant KM, et al. Incidence of thrombotic complications in critically ill ICU patients with COVID-19. Thromb Res. 2020 Jul;191:145-7.

4 Ackermann M, Verleden SE, Kuehnel M, Haverich A, Welte T, Laenger F, et al. Pulmonary vascular endothelialitis, thrombosis, and angiogenesis in Covid-19. N Engl J Med. 2020;383(2):120-8. 
5 Nicolai L, Leunig A, Brambs S, Kaiser R, Weinberger $\mathrm{T}$, Weigand $\mathrm{M}$, et al. Immunothrombotic dysregulation in COVID-19 pneumonia is associated with respiratory failure and coagulopathy. Circulation. 2020 Sep 22;142(12):1176-89.

6 Levi M, Thachil J, Iba T, Levy JH. Coagulation abnormalities and thrombosis in patients with COVID-19. Lancet Haematol. 2020 Jun; 7(6):e438-e40.

7 Cavalcanti DD, Raz E, Shapiro M, Dehkharghani S, Yaghi S, Lillemoe K, et al. Cerebral venous thrombosis associated with $\mathrm{CO}$ VID-19. Am J Neuroradiology. 2020 Aug; 41(8):1370-76.

8 Cui S, Chen S, Li X, Liu S, Wang F. Prevalence of venous thromboembolism in patients with severe novel coronavirus pneumonia. J Thromb Haemost. 2020 Jun; 18(6):1421-4.

9 Miesbach W, Makris M. COVID-19: coagulopathy, risk of thrombosis, and the rationale for anticoagulation. Clin Appl Thromb Hemost. 2020 Jan-Dec;26:1076029620938149.

10 Paranjpe I, Fuster V, Lala A, Russak AJ, Glicksberg BS, Levin MA, et al. Association of treatment dose anticoagulation with in-hospital survival among hospitalized patients with COVID-19. J Am Coll Cardiol. 2020 Jul 7;76(1):122-4.

11 Kanitra JJ, Power AD, Hayward RD, Haouilou JC, Edhayan E. Malfunctioning temporary hemodialysis catheters in patients with COVID-19. J Vasc Surg. 2021 Jun;73(6): 1881-8.

12 Helms J, Tacquard C, Severac F, Leonard-Lorant I, Ohana M, Delabranche X, et al. High risk of thrombosis in patients with severe SARS-CoV-2 infection: a multicenter prospective cohort study. Intensive Care Med. 2020 Jun;46(6):1089-98.

13 Sise ME, Baggett MV, Shepard JO, Stevens JS, Rhee EP. Case 17-2020: a 68-year-old man with Covid-19 and acute kidney injury. N Engl J Med. 2020 May 28;382(22):2147-56.
14 Parienti JJ, Mégarbane B, Fischer MO, Lautrette A, Gazui N, Marin N, et al. Catheter dysfunction and dialysis performance according to vascular access among 736 critically ill adults requiring renal replacement therapy: a randomized controlled study. Crit Care Med. 2010 Apr;38(4):1118-25.

15 Benichou N, Lebbah S, Hajage D, MartinLefèvre L, Pons B, Boulet E, et al. Vascular access for renal replacement therapy among 459 critically ill patients: a pragmatic analysis of the randomized AKIKI trial. Ann Intensive Care. 2021 Apr 8;11(1):56.

16 Brosnahan SB, Smilowitz NR, Amoroso NE, Barfield M, Berger JS, Goldenberg R, et al. Thrombosis at hospital presentation in patients with and without coronavirus disease 2019. J Vasc Surg Venous Lymphat Disord. 2021 Jul;9(4):845-52.

17 Fournier M, Faille D, Dossier A, Mageau A, Nicaise Roland P, Ajzenberg N, et al. Arterial thrombotic events in adult inpatients with COVID-19. Mayo Clin Proc. 2021 Feb;96(2): 295-303.

18 Ilonzo N, Rao A, Safir S, Vouyouka A, Phair J, Baldwin M, et al. Acute thrombotic manifestations of coronavirus disease 2019 infection: experience at a large New York City health care system. J Vasc Surg. 2021 Mar; 73(3):789-96.

19 Desbuissons G, Michon A, Attias P, Burbach M, Diaconita M, Karie-Guiges S, et al. Arteriovenous fistulas thrombosis in hemodialysis patients with COVID-19. J Vasc Access. 2021 Feb 24:1129729821996091.

20 Gupta S, Wang W, Hayek SS, Chan L, Mathews KS, Melamed ML, et al. Association between early treatment with tocilizumab and mortality among critically Ill patients with COVID-19. JAMA Intern Med. 2021 Jan 1; 181(1):41-51.
21 Chang Y, Ryu S, Choi Y, Zhang Y, Cho J, Kwon MJ, et al. Metabolically healthy obesity and development of chronic kidney disease: a cohort study. Ann Intern Med. 2016 Mar 1; 164(5):305-12.

22 Danziger J, Chen KP, Lee J, Feng M, Mark RG, Celi LA, et al. Obesity, acute kidney injury, and mortality in critical illness. Crit Care Med. 2016 Feb;44(2):328-34.

23 Gameiro J, Gonçalves M, Pereira M, Rodrigues N, Godinho I, Neves M, et al. Obesity, acute kidney injury and mortality in patients with sepsis: a cohort analysis. Ren Fail. 2018 Nov;40(1):120-6.

24 Gerotziafas GT, Sergentanis TN, Voiriot G, Lassel L, Papageorgiou C, Elabbadi A, et al. Derivation and validation of a predictive score for disease worsening in patients with COVID-19. Thromb Haemost. 2020 Dec: 120(12):1680-90.

25 Paliogiannis P, Mangoni AA, Cangemi M, Fois AG, Carru C, Zinellu A. Serum albumin concentrations are associated with disease severity and outcomes in coronavirus 19 disease (COVID-19): a systematic review and metaanalysis. Clin Exp Med. 2021 Aug;21(3):34354.

26 Wiedermann CJ, Wiedermann W, Joannidis M. Causal relationship between hypoalbuminemia and acute kidney injury. World J Nephrol. 2017 Jul 6;6(4):176-87.

27 Folsom AR, Lutsey PL, Heckbert SR, Cushman M. Serum albumin and risk of venous thromboembolism. Thromb Haemost. 2010 Jul;104(1):100-4.

28 Basili S, Carnevale R, Nocella C, Bartimoccia S, Raparelli V, Talerico G, et al. Serum albumin is inversely associated with portal vein thrombosis in cirrhosis. Hepatol Commun. 2019 Apr;3(4):504-12.

29 Violi F, Ceccarelli G, Loffredo L, Alessandri F, Cipollone F, D'Ardes D, et al. Albumin supplementation dampens hypercoagulability in COVID-19: a preliminary report. Thromb Haemost. 2021 Jan;121(1):102-5. 